\title{
Insights Into Peptoid Helix Folding Cooperativity From an Improved Backbone Potential
}

Sudipto Mukherjee, Guangfeng Zhou, Chris Michel ${ }^{\dagger}$ and Vincent A. Voelz*

Department of Chemistry, Temple University, Philadelphia PA

* corresponding author: voelz@ temple.edu

p. 2-4 Supporting Tables S1-S4.

p. 5-8 Supporting Figures S1-S4. 
Table S1. Trajectory lengths of the simulation data in this study

\begin{tabular}{lll} 
Chain length & \multicolumn{2}{l}{ simulation length $(\mu \mathrm{s})$} \\
& GAFF- $\phi$ & GAFF \\
\hline 3 & 6.14 & 4.00 \\
4 & 4.42 & 4.00 \\
5 & 3.47 & 4.00 \\
6 & 4.23 & 4.00 \\
7 & 3.92 & 4.00 \\
8 & 5.12 & 4.00 \\
9 & 4.20 & 4.00 \\
10 & 4.14 & 4.00 \\
11 & 4.00 & 4.00 \\
12 & 3.95 & 3.05 \\
13 & 3.95 & 2.77 \\
14 & 3.95 & 2.72 \\
15 & 3.89 & 4.00 \\
\hline average & 4.26 & 3.73 \\
total & 55.38 & 48.53
\end{tabular}


Table S2. Per-residue $\omega$-angle autocorrelation times for Ac-(Nspe $)_{5}-\mathrm{NH}_{2}$, calculated from singleexponential fits to the autocorrelation function results shown in Figure S2.

residue autocorrelation time $\tau(\mathrm{ns})$

\begin{tabular}{ll}
\hline 1 & 13.47 \\
2 & 45.93 \\
3 & 98.90 \\
4 & 86.81 \\
5 & 16.09
\end{tabular}

Table S3. BICePs-computed Bayes factors $P\left(M_{1}\right) / P\left(M_{2}\right)$ comparing models $M_{1}=$ GAFF- $\phi+\exp$ and $M_{2}=\mathrm{GAFF}+\exp$, using various experimental $K_{\mathrm{ct}}$ values.

$K_{\text {ct }} \quad$ Bayes Factor

$1.5 \quad 1.32$

$1.9 \quad 1.54$

$2.2 \quad 1.51$

$2.5 \quad 1.53$

$2.8 \quad 1.45$

$3.2 \quad 1.69$ 
Table S4. A comparison of the ten largest conformational populations of (Nspe $)_{5}$ calculated using the BICePs algorithm, for GAFF- $\phi+$ exp model, and GAFF + exp. Strings describing backbone conformations denote the pattern of $\omega$ (cis (c) vs. trans (t)) and $\phi$ angles (positive or negative).

\begin{tabular}{llll} 
Model & Population & State index & Backbone conformation \\
\hline \multirow{3}{*}{ GAFF- $\phi+\exp$} & 0.458 & 623 & $c-c-c-c-t-$ \\
& 0.138 & 716 & $c-c-c-c-t-$ \\
& 0.0907 & 369 & $c-c-c-c-t-$ \\
& 0.0548 & 553 & $c-c-c-c-t-$ \\
& 0.0465 & 606 & $c-c-c-c-t-$ \\
& 0.0279 & 624 & $c-c-c-c-t-$ \\
& 0.0217 & 596 & $c-c-c-c-t-$ \\
& 0.0215 & 536 & $c-c-c-c-t-$ \\
total & 0.0131 & 248 & $c-c-c-c-t-$ \\
& 0.0087 & 923 & $c-c-c-c-t-$ \\
GAFF $+\exp$ & $\mathbf{0 . 8 8 2}$ & & \\
& 0.243 & 644 & $c-c+t+c-t+$ \\
& 0.1366 & 957 & $c-c+t+c-t+$ \\
& 0.1082 & 578 & $c-c-c-c+t+$ \\
& 0.0639 & 632 & $c-c-c-t+t+$ \\
& 0.0464 & 422 & $c+c+t-t+t+$ \\
& 0.0349 & 666 & $c-c+t+c-t-$ \\
& 0.0262 & 189 & $c-c-c-c+t+$ \\
& 0.0245 & 850 & $c+c+t-c+t-$ \\
& 0.0214 & 820 & $t+c+c+c+t+$ \\
& 0.0207 & 887 & $c-c+t+c-t-$ \\
\cline { 2 - 4 } & $\mathbf{0 . 7 2 6}$ & &
\end{tabular}



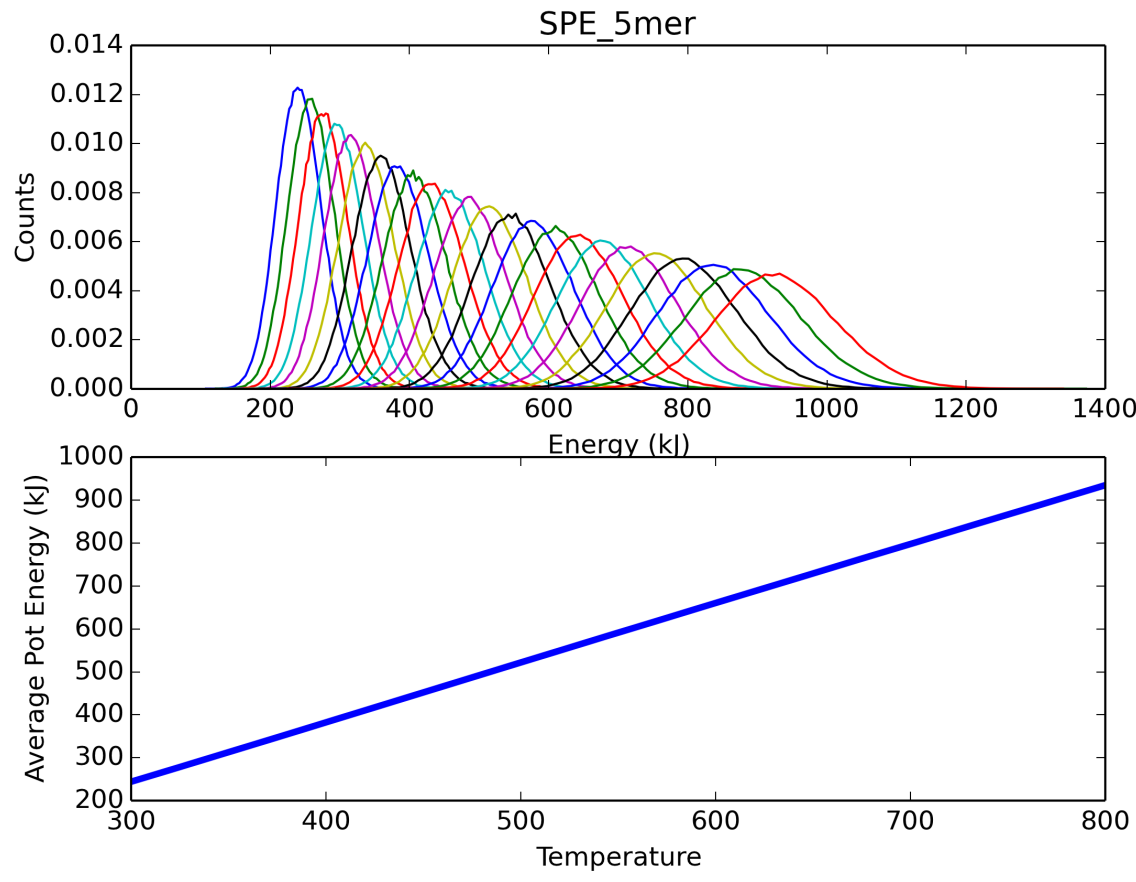

Figure S1. Potential energy distributions all 24 simulation replicas of Ac-(Nspe $)_{5}-\mathrm{NH}_{2}$ show excellent overlap, ensuring replica exchange acceptance ratios of $>0.5$. Similar acceptance ratios were achieved for all chain lengths simulated. Simulation temperatures used were: 300 , $313,327,341,356,371,387,404,422,440,460,480,500,522,545,569,594,619,646,675$, $704,735,767,800 \mathrm{~K}$. 


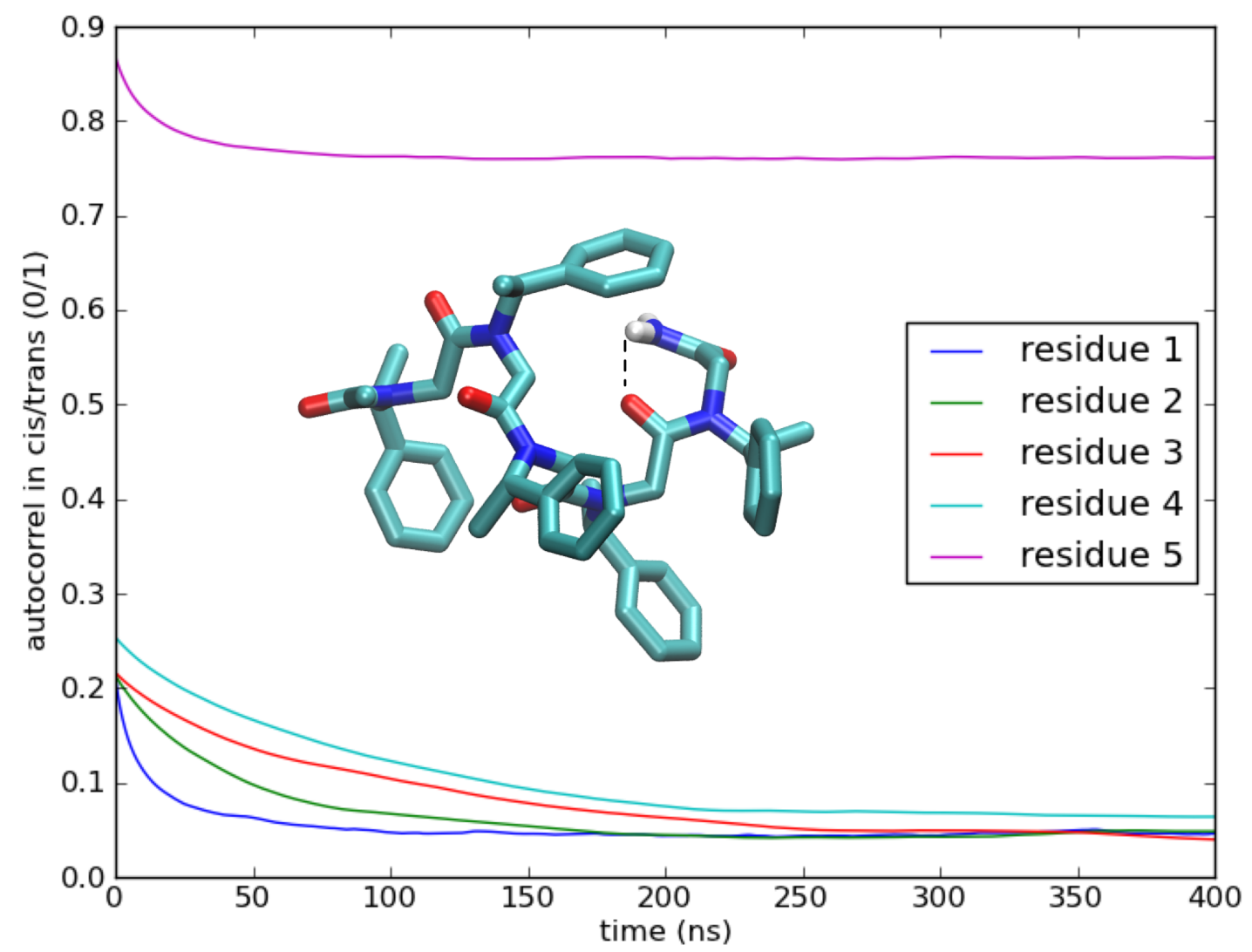

Figure S2. Autocorrelation times for $\omega$ angles in molecular simulation trajectories of Ac(Nspe) $)_{5}-\mathrm{NH}_{2}$. Line plots show the autocorrelation functions $g(t)=\left\langle\chi\left(t^{\prime}\right) \chi\left(t^{\prime}+t\right)\right\rangle$ for each residue's $\omega$ angle, where $\chi(\omega)$ is an indicator function equal to zero for cis $\omega$ angles $\left(|\omega|<90^{\circ}\right)$ and equal to one for trans $\omega$ angles $\left(|\omega|>+90^{\circ}\right)$. Single-exponential fits to these results yield the autocorrelation time values shown in Table S2. 
gaff-phi
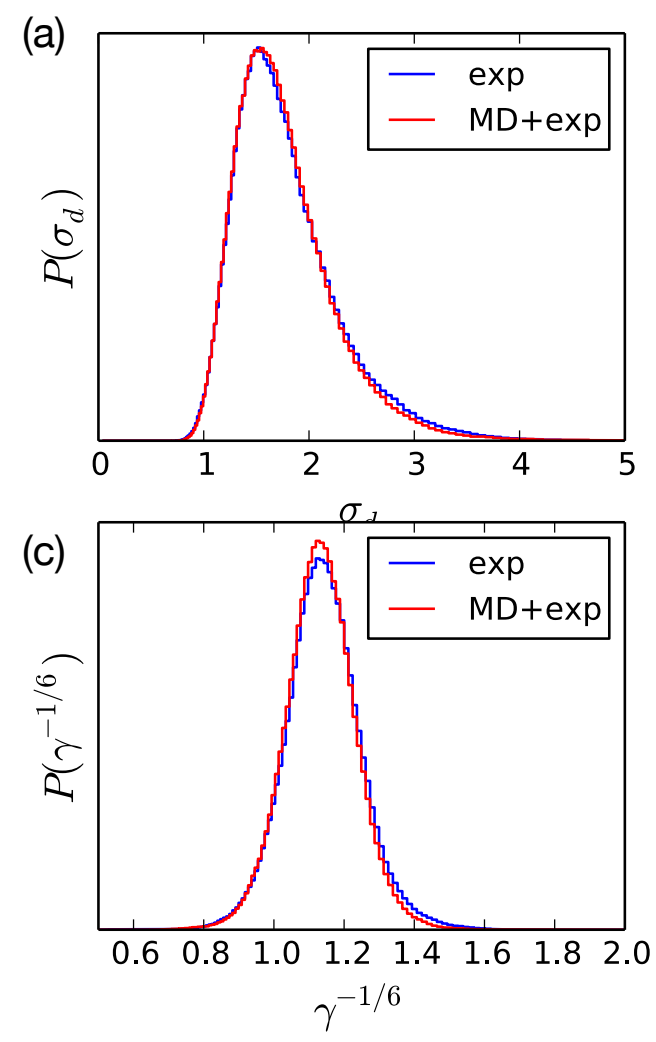

gaff
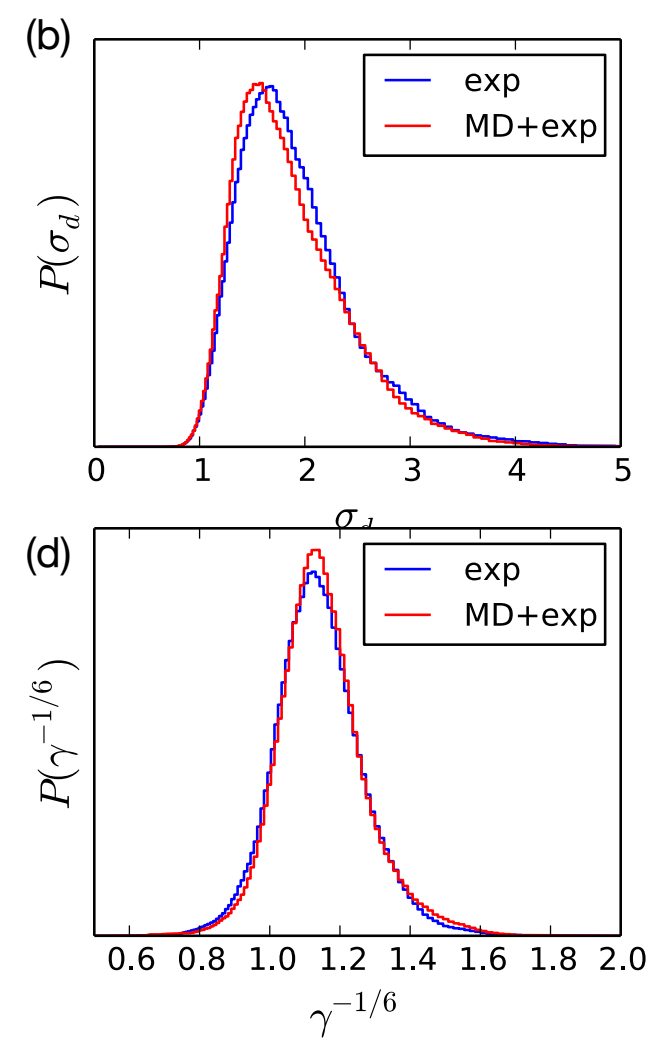

Figure S3. Posterior distributions of nuisance parameters $\sigma$ sampled by the BICePs algorithm for (a) GAFF- $\phi+\exp$ and (b) GAFF + exp. Posterior distributions of nuisance parameters $\gamma^{\prime}$ for (c) GAFF- $\phi+\exp$ and (d) GAFF + exp. 


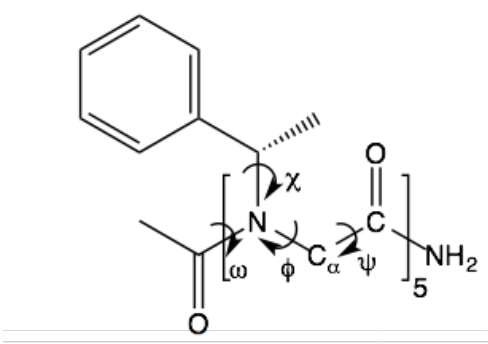

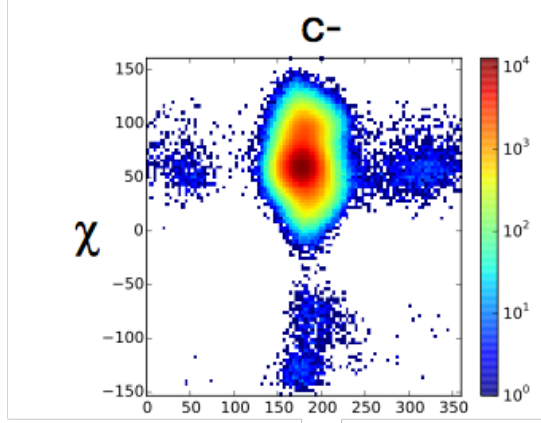

$\psi$

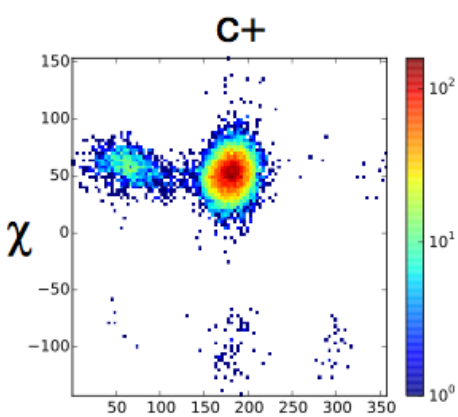

$\psi$

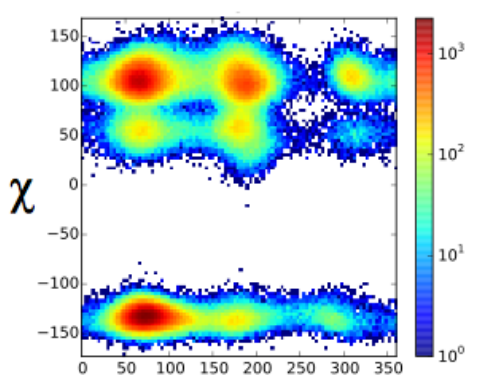

$\psi$

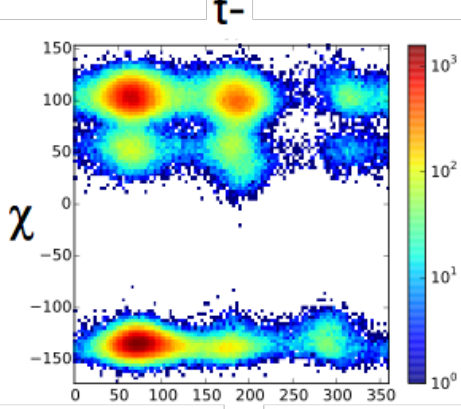

$\psi$

Figure S4. Distributions of $\chi$ and $\psi$ angles in all four helical states of the backbone, 'c-', 'c+', ' $t$-' and ' $t+$ ' observed in the lowest-temperature replica of a 3.4 REMD simulation of Ac(Nspe) $)_{5}-\mathrm{NH}_{2}$. Shown the average across all residues. 